\title{
Macular Hole Surgery in a Patient Who Cannot Maintain Facedown Positioning
}

\author{
Zofia Michalewska Jerzy Nawrocki \\ Ophthalmic Clinic 'Jasne Blonia', Lodz, Poland
}

Key Words

Macular hole $\cdot$ Inverted ILM flap $\cdot$ SD-OCT

\begin{abstract}
Aim: To present macular hole surgery in a patient who had previously undergone thyroid removal surgery.
\end{abstract}

Material and Methods: During thyroid gland removal surgery, the recurrent laryngeal nerves were cut by the surgeon. Therefore, the patient had to have a tracheotomy and because of this unusual situation, the patient could not breathe if lying 'upside-down'. Complete ophthalmic examination and spectral optical coherence tomography was performed in a 77year-old woman before and after macular hole surgery.

Results: The patient was treated by the 'inverted internal limiting membrane (ILM) flap technique' with air tamponade for macular hole closure. This technique was described to have very high success rates in large, stage IV macular holes. Postoperatively, lying on her opposite side was advised. However, the macular hole remained open after this approach. Because of this, another approach was undertaken. The ILM flap technique and silicone oil were applied, and the patient was positioned on her opposite side. Silicone oil was removed after 3 months. Eighteen months later, the macular hole remained closed. Visual acuity improved from $10 / 200$ to $20 / 50$.

Conclusion: This case demonstrates that in an extremely select group of patients, silicone oil combined with the inverted flap technique may be considered for treatment of macular hole.

\section{Background}

Idiopathic macular hole is a macular disease affecting mostly women after the 5th decade. Before 1991, the disease was thought to be untreatable until Kelly and Wendel [1] reported over $60 \%$ success after pars plana vitrectomy. Nowadays, due to new surgical equipment and diagnostic tools, the success rate after macular hole surgery is

Zofia Michalewska, MD, PhD 
reported to be about $90-98 \%[2,3]$. Although some authors reported satisfactory results without postoperative prone positioning [4], most surgeons still advise it. However, in cases of long-lasting macular holes with a diameter of more than $400 \mu \mathrm{m}$, optical coherence tomography (OCT) and spectral-domain (SD)-OCT data demonstrate that $19-39 \%$ of so-called 'closed macular holes' have, in reality, flat borders but bare retinal pigment epithelium (flat-open macular holes) [5-7]. In these eyes, visual acuity is usually limited $(0.02-0.2)$.

For large macular holes, different techniques have been proposed to improve surgical outcomes [8-10]. Recently, a prospective randomized study presented by our group proved that the inverted internal limiting membrane (ILM) flap (inverted ILM flap) technique' improves functional and anatomic results in patients with large macular holes as compared with pars plana vitrectomy with ILM peeling and air tamponade. Functional outcomes were also better than those presented in earlier nonrandomized studies on silicone oil injection or mechanical bringing together of the borders of the macular hole [11].

Herein, we report a single case of failure of the inverted flap technique in a patient after tracheotomy.

\section{Material and Methods}

\section{Case Report}

A 77-year-old pseudophakic patient presented to our clinic with a macular hole. The patient reported decreased visual acuity lasting more than 12 months. The upper diameter of the macular hole was $400 \mu \mathrm{m}$, the lower diameter $1,617 \mu \mathrm{m}$, and posterior hyaloid traction was visible (fig. 1a). Initial visual acuity was 0.04 . Six years earlier, the patient had had thyroid gland cancer, and during surgery the recurrent pharyngeal nerves were cut. The patient had subsequent tracheotomy. Due to this situation, the patient was not able to breathe if lying 'upside down' and could not maintain facedown positioning after macular hole surgery. Because of the large size of the macular hole, the patient was advised to have macular hole surgery with the inverted ILM flap technique [11].

This technique consists of core vitrectomy and trypan blue-assisted ILM peeling. During the circumferential peeling, the ILM was not removed completely from the retina but was left attached to the edges of the macular hole. A rolled segment of the peeled ILM was hanging in the vitreous cavity. Next, a peripheral piece of the ILM was trimmed with a vitreous cutter or vitreous scissors and the central part of the ILM was left in place. Consequently, small remnants of the ILM remained surrounding the macular hole. The ILM was then massaged gently over the macular hole from all sides until the ILM became inverted, or upside down, such that the surface that normally faces the vitreous body now faced the retinal pigment epithelium. The macular hole was covered with the inverted ILM flap [11]. At the end of surgery, the vitreous cavity was filled with air. The patient was advised to lie on the opposite side.

The macular hole did not close either 1 week or 1 month after surgery (fig. 1b), and the patient qualified for repeat surgery with silicone oil. This was the first case of failure after the use of the inverted flap technique, even though the borders of the macular hole were still elevated and the lower diameter shortened to $1,494 \mu \mathrm{m}$. On the inner surface of the retina, a delicate hyperreflective structure was visible. Its reflectivity and thickness were lower than epiretinal membranes usually have.

During the second procedure, it was observed that the ILM flap was lying on the retinal surface instead of being inverted. The rolled segment of the peeled ILM, which was lying on the retinal surface, was massaged gently over the macular hole from all sides until the ILM became inverted. The vitreous cavity was filled with silicone oil. The patient was advised to lie on the opposite side. 
The macular hole was closed 1 week after surgery (fig. 1c). However, a hyporeflective area was visible between the outer retinal layers and retinal pigment epithelium for about 10 months after surgery. The macular hole remained closed after silicone oil removal.

Visual acuity was 0.15 six months after surgery (fig. 1d) and then gradually improved to 0.4 eighteen months after surgery (fig. 1e). During that time, SD-OCT images revealed that the macula gradually became filled with tissue. During the final examination, macular contour seemed almost normal. Small defects of the junction between outer and inner segments of the photoreceptors and external limiting membrane remained paracentral to the fovea. On the inner retinal surface, a delicate hyperreflective structure can be seen even 18 months after surgery, without any evidence of epiretinal membrane formation.

\section{Discussion}

Large, long-standing macular holes are less likely to be closed after pars plana vitrectomy. The inverted ILM flap technique was recently reported to be successful in nearly $100 \%$ of patients with macular holes with a diameter of more than $400 \mu \mathrm{m}$. The single failures were observed in eyes in which it was impossible to perform the inverted ILM technique due to the fact that the inverted ILM flap became torn off the retinal surface during surgical maneuvers.

This case presents a failure of this technique, even though the procedure was correctly performed and the inverted ILM flap was observed on postoperative SD-OCT images and during the secondary procedure. The most probable cause of failure was the fact that the patient was unable to maintain facedown positioning due to a previous tracheotomy.

The authors assumed that lying on the side might have been enough to obtain closure of the macular hole, but it was not. The ILM flap was inverted during surgery, but changed its position, probably during fluid/air exchange. Postoperative SD-OCT images showed the ILM flap lying on the retinal surface. Also, during the secondary procedure it was noted and re-inverted. The position of the inverted ILM flap is much more controllable under silicone oil than fluid/air exchange.

Even though a high success rate was reported by some authors in patients who were not advised to maintain facedown positioning, it must be mentioned that those papers mostly describe results of surgery in stage I or II macular holes. This may suggest that air/gas tamponade may be avoided in smaller macular holes, but it is crucial for the success of large, stage IV macular holes. It may also be true that air endotamponade is crucial for the success of the inverted ILM flap technique. One possible mechanism of explaining air/gas tamponade is that the bubble is a scaffold for cell migration to close the macular hole. Another hypothesis is that the bubble separates retinal pigment epithelium from fluid.

It was documented by SD-OCT that in most cases macular holes close during the first days after surgery [12]. However, in some cases the closure process in prolonged, especially in large macular holes [13]. Some authors use silicone oil as endotamponade in large, stage IV macular holes, especially in secondary procedures. In this paper, we present for the first time the use of silicone oil tamponade as an adjunct to the inverted flap technique. It may be observed that beneath the silicone oil, the fragments of ILM cover the macular defect. 
To conclude, this report suggests that postoperative prone positioning is an important criterion for closure of large macular holes. Silicone oil may be advised in some selected cases as an adjunct to the inverted ILM flap technique. Additionally, postoperative improvement of visual acuity and retinal architecture may last many months after macular hole surgery.

\section{Disclosure Statement}

The authors declare no proprietary interests and no funding was received in support of this study. 

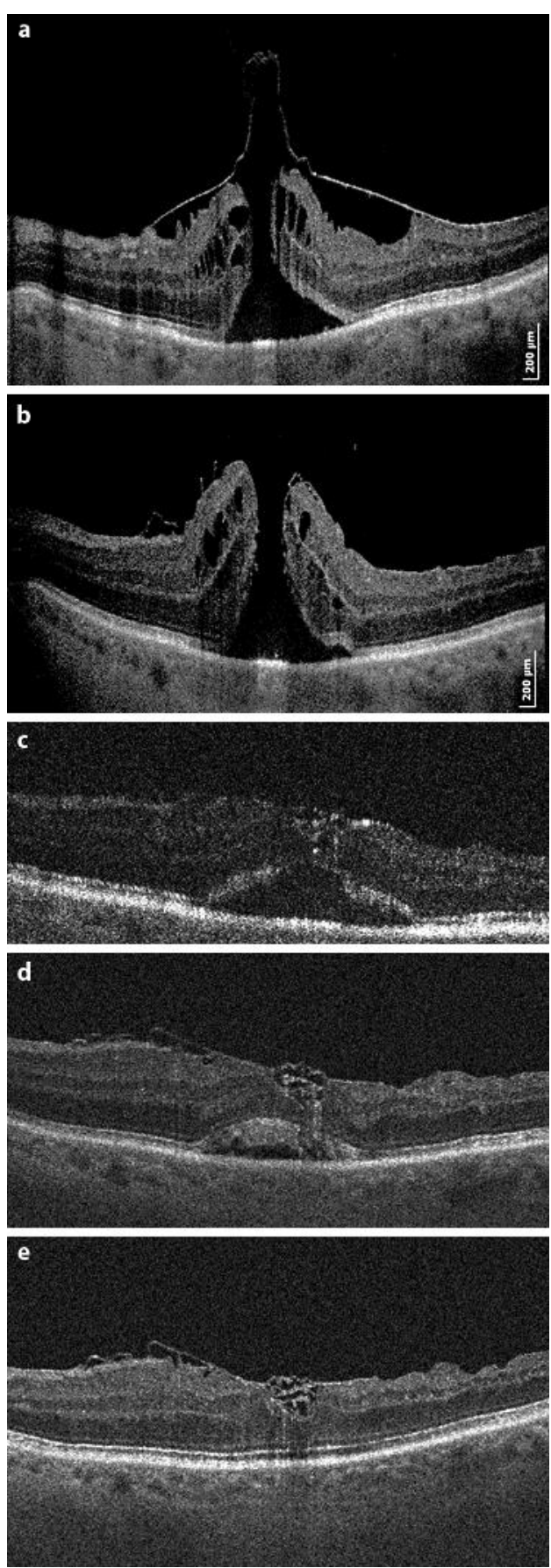

Fig. 1. a Full-thickness, stage IV macular hole. Inner diameter is $400 \mu \mathrm{m}$. Outer diameter is $1,617 \mu \mathrm{m}$. Vitreofoveal traction is visible. Visual acuity is 0.04 . b Full-thickness macular hole after the first surgery. Remnants of the inverted ILM flap are visible on the retinal surface. They do not cover the macular hole. c One week after vitrectomy with silicone oil. The macular hole is closed, but the fovea contour is irregular and a cystoid space in the outer retina is visible. $\mathbf{d}$ One week after silicone oil removal. The line representing the junction between inner and outer segments of photoreceptors tends to normalize. Outer photoreceptor segments seem elongated. e Fovea appearance 18 months after surgery. Fovea contour is almost normal. Remnants of the ILM are still visible on retinal surface. The line representing the junction between inner and outer segments of photoreceptors seems intact in the fovea. Visual acuity is 0.4 . 


\section{References}

1 Kelly NE, Wendel RT: Vitreous surgery for idiopathic macular holes: results of a pilot study. Arch Ophthalmol 1991;109:654-659.

-2 Ando F, Sasano K, Ohba N, et al: Anatomic and visual outcomes after indocyanine green-assisted peeling of the retinal internal limiting membrane in idiopathic macular hole surgery. Am J Ophthalmol 2004;137:609-614.

-3 Beutel J, Dahmen G, Ziegler A, Hoerauf H: Internal limiting membrane peeling with indocyanine green or trypan blue in macular hole surgery: a randomized trial. Arch Ophthalmol 2007;125:326-332.

4 Holekamp N, Tornambe P: Surgery for macular hole repair: a conversation. Is facedown positioning necessary? Retina Today 2011;3:80-84.

-5 Michalewska Z, Michalewski J, Cisiecki S, Nawrocki J: High speed, high resolution spectral optical coherence tomography after macular hole surgery. Graefes Arch Clin Exp Ophthalmol 2008;246:823830.

-6 Imai M, Iijima H, Gotoh T, Tsukahara S: Optical coherence tomography of successfully repaired idiopathic macular holes. Am J Ophthalmol 1999;128:621-627.

>7 Kang SW, Ahn K, Ham DI: Types of macular hole closure and their clinical implications. Br J Ophthalmol 2003;87:1015-1019.

-8 Alpatov S, Shchuko A, Malyshev V: A new method of treating macular holes. Eur J Ophthalmol 2007;17:246-252.

-9 Couvillion SS, Smiddy WE, Flynn HW Jr, Eifrig CWG, Gregori G: Outcome of surgery for idiopathic macular hole: a case-control study comparing silicone oil with gas tamponade. Ophthalmic Surg Lasers Imaging 2005;36:365-371.

10 Goldbaum MH, McCuen BW, Hanneken AM, Burgess SK, Chen HH: Silicone oil tamponade to seal macular holes without position restrictions. Ophthalmology 1998;105:2140-2148.

-11 Michalewska Z, Michalewski J, Adelman RA, Nawrocki J: Inverted internal limiting membrane (ILM) flap technique for large macular hole. Ophthalmology 2010;117:2018-2025.

-12 Jumper JM, Gallemore RP, McCuen BW 2nd, Toth CA: Features of macular hole closure in the early postoperative period using optical coherence tomography. Retina 2000;20:232-237.

13 Michalewska Z, Michalewski J, Nawrocki J: Continuous changes in macular morphology after macular hole closure visualized with spectral optical coherence tomography. Graefes Arch Clin Exp Ophthalmol 2010;248:1249-1255. 